\title{
Relationship between abdominal obesity and alcohol drinking pattern in normal-weight, middle-aged adults: the Korea National Health and Nutrition Examination Survey 2008-2013
}

\author{
Kye-Yeung Park, Hoon-Ki Park* and Hwan-sik Hwang \\ Department of Family Medicine, Hanyang University Medical Centre, 222 Wangsimni-ro, Seongdong-gu, \\ Seoul 04763, Republic of Korea
}

Submitted 9 October 2016: Final revision received 10 March 2017: Accepted 24 April 2017: First published online 23 June 2017

\begin{abstract}
Objective: Abdominal obesity is associated with obesity-related health risks regardless of body weight. The present study aimed to determine whether alcohol drinking pattern is associated with abdominal obesity in normal-weight, middle-aged adults. Design: Cross-sectional study using complex sampling design analyses.

Setting: The Korea National Health and Nutrition Examination Survey, which was conducted from 2008 to 2013.

Subjects: Normal-weight participants aged 40-69 years with BMI of $18 \cdot 5-25 \cdot 0 \mathrm{~kg} / \mathrm{m}^{2}$ ( $n$ 11289, 4491 men and 6798 women) were included. Abdominal obesity was defined as waist circumference $\geq 90 \mathrm{~cm}$ for men or $\geq 85 \mathrm{~cm}$ for women. Alcohol drinking pattern was assessed by self-report on questionnaires.

Results: Among 11289 normal-weight participants, 7.9\% ( $n$ 379) of men and $7 \cdot 6 \%$ ( $n$ 609) of women had abdominal obesity. Both men and women who consumed a higher quantity of alcohol per drinking occasion had higher odds (OR; 95\% CI) for abdominal obesity compared with individuals who consumed fewer than 2 drinks (1.86; 1.04, 3.32 for $\geq 10$ drinks/typical occasion in men; and 3.28; 1.13, 9.46 in women). Men who binge drink every day had higher odds for abdominal obesity $(2 \cdot 10 ; 1 \cdot 21,3.63)$. In both sexes, frequency of alcohol drinking was not associated with normal-weight abdominal obesity.

Conclusions: Our study showed that the amount of alcohol drinking per occasion influenced abdominal obesity in normal-weight, middle-aged individuals that may have impacted obesity-related health risks. Healthy alcohol drinking habits need to be controlled for prevention of abdominal obesity even among persons with normal weight.
\end{abstract}

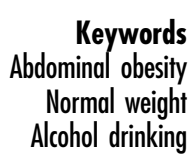

Obesity is defined as a medical condition of abnormal or excessive fat accumulation that may impair health ${ }^{(1)}$. With the growing obesity epidemic, it is recommended that physicians assess and manage obesity through appropriate measurements in clinical practice for the assessment of health risk ${ }^{(2,3)}$. Anthropometric parameters for the diagnosis of obesity include BMI as an indicator of general obesity and waist circumference (WC) or waist-to-hip ratio (WHR) as an indicator of abdominal adiposity ${ }^{(4-6)}$. BMI reflects lean body mass as well as fat mass and does not consider fat distribution ${ }^{(7)}$. Compared with BMI, indices of abdominal adiposity such as WC and WHR provide more information about the central distribution of body fat ${ }^{(5)}$. There is some evidence from prospective cohort studies and metaanalyses that abdominal obesity measures such as WC or WHR are associated with obesity-related mortality in both men and women, regardless of general obesity ${ }^{(6,8,9)}$.
A recent large cohort study identified that normal-weight adults with central obesity (identified by WHR) had a higher rate of mortality compared with participants with normal fat distribution, regardless of $\mathrm{BMI}^{(10)}$. Another study showed that individuals with a high body fat content were associated with a higher prevalence of insulin resistance, altered lipid profile, metabolic syndrome and cardiovascular risk factors, despite a normal $\mathrm{BMI}^{(11)}$. One longitudinal study has revealed that the presence of abdominal obesity predicts insulin resistance, especially in normal-weight individuals, thereby increasing the risks of diabetes and $\mathrm{CVD}^{(12)}$.

Abdominal obesity occurs in the context of a variety of changes in dietary intake, physical activity, and socioeconomic and lifestyle factors ${ }^{(13)}$. Alcohol consumption appears to have become an important lifestyle element in both Western and Asian countries, and there is growing interest in better understanding its health risks ${ }^{(14)}$. 
Previous studies examining the association between alcohol consumption and abdominal obesity have shown inconsistent results ${ }^{(15)}$. Several studies have reported a positive association between alcohol drinking and abdominal obesity in heavy alcohol consumption ${ }^{(16)}$, and even moderate alcohol consumption ${ }^{(17)}$; other studies have found that the amount or intensity of drinking is positively correlated with abdominal obesity, while the frequency of drinking is negatively correlated ${ }^{(18)}$. Binge drinking was associated with an increased likelihood of being obese in one study ${ }^{(19)}$. A cohort study showed that alcohol intake was not associated with 5-year difference in WC in either women or men ${ }^{(20)}$.

To the best of our knowledge, data on the association between alcohol drinking pattern and abdominal obesity in normal-weight persons are limited. One study has reported that low to moderate alcohol consumption appears to be inversely associated with future weight and WC in middleaged adults ${ }^{(21)}$. Likewise, middle-aged adults, a group at high risk for cardiovascular and other chronic conditions including obesity, are exposed to various potential health problems due to lifestyle factors such as physical activity, diet, alcohol drinking and smoking ${ }^{(22)}$. The theoretical rationale for the present study is based on these considerations. We hypothesized that there exists a specific positive relationship between each of various measures of alcohol drinking pattern (alcohol drinking frequency, alcohol drinking amount, frequency of binge drinking, total alcohol intake) and abdominal obesity in normal-weight, middle-aged persons. Therefore, the present study aimed to examine the relationship between three variables of alcohol drinking pattern (alcohol drinking frequency, alcohol drinking quantity, binge drinking) and abdominal obesity (as defined by WC) among the population of normal-weight individuals aged 40-69 years old in Korea.

\section{Methods}

\section{Study population}

The current cross-sectional study was conducted using data collected by the Korea National Health and Nutrition Examination Survey (KNHANES), which has been conducted annually since 1998 by the Korean Ministry of Health and Welfare. The survey was designed to assess the health and nutrition status of the non-institutionalized civilian population of South Korea and has three components: a health interview survey, a health examination survey and a nutrition survey. To increase the power of the analyses, we combined data from three separate waves of the survey (2008-2009, 2010-2012 and 2013) ${ }^{(23)}$. The 2008-2013 KNHANES investigated populations of approximately 10000 individuals from twenty households in 200 enumeration districts per year based on a threestage sampling method (household, enumeration districts and local districts). It is a complex, stratified, multistage, cluster-sampling method with proportional allocation based on geographic area, sex and age. Participants for the current study were normal-weight, middle-aged adults. Among 33738 participants aged 20 years or older, we excluded participants aged less than 40 years or above 69 years $(n$ 15450) and those with missing data for BMI ( $n$ 63) or WC ( $n$ 30). The final study sample consisted of 18195 participants, which included 403 participants with BMI lower than $18.5 \mathrm{~kg} / \mathrm{m}^{2}, 11289$ participants with BMI of 18.5 to $25.0 \mathrm{~kg} / \mathrm{m}^{2}$, and 6503 participants with BMI of $25.0 \mathrm{~kg} / \mathrm{m}^{2}$ or more. Therefore, for the present study, a total of 11289 participants (4491 men; 6798 women) were included in the analysis of the association between alcohol consumption and abdominal obesity in normal-weight, middle-aged adults.

\section{Definition of abdominal obesity and obesity}

Anthropometric measurements of participants were performed by well-trained examiners. Body weight and height were measured while participants wore light clothing and were barefoot. BMI was calculated as weight divided by the square of height $\left(\mathrm{kg} / \mathrm{m}^{2}\right)$. According to the Asia-Pacific guidelines, BMI $<18.5 \mathrm{~kg} / \mathrm{m}^{2}$ was regarded as underweight and $\mathrm{BMI} \geq 25 \cdot 0 \mathrm{~kg} / \mathrm{m}^{2}$ was used to define obesity ${ }^{(24)}$. WC was measured at the midline between the lowest costal rib and the uppermost iliac crest in the mid-axillary line, with the participant standing. The cut-off point for WC for abdominal obesity in Koreans was defined as $90 \mathrm{~cm}$ for men and $85 \mathrm{~cm}$ for women ${ }^{(25)}$. Therefore, individuals with normal-weight abdominal obesity in our study were those with BMI of $18 \cdot 5-25 \cdot 0 \mathrm{~kg} / \mathrm{m}^{2}$ and WC $\geq 90 \mathrm{~cm}$ for men or $\geq 85 \mathrm{~cm}$ for women.

\section{Assessment of alcobol drinking patterns}

Alcohol consumption patterns during the previous year were recorded using self-reported questionnaires, which included typical drinking quantity per drinking occasion (1-2 drinks, 3-4 drinks, 5-6 drinks, 7-9 drinks, $\geq 10$ drinks), average drinking frequency ( $<1 /$ month, $1 /$ month, $2-4$ /month, 2-3/week, $\geq 4$ week) and frequency of binge drinking (none, $<1$ /month, $1 /$ month, 1 /week, every day). In these questions, a standard 'drink' was defined as a single glass of liquor, wine or the Korean traditional drink So-ju (Korean distilled liquor). One bottle of beer $(355 \mathrm{ml})$ was counted as 1.6 standard drinks. Binge drinking was defined as consuming $\geq 7$ standard drinks ( $\geq 5$ drinks for women) consecutively on one occasion ${ }^{(26)}$. To yield an average measure of total alcohol intake, the categories of typical drinking quantity and average drinking frequency were converted into number, and the intake was categorized into four groups ( $<7$ drinks/week, 7-13 drinks/ week, 14-20 drinks/week, $\geq 21$ drinks/week) ${ }^{(18)}$.

\section{Measurement of covariates}

Each participant was interviewed using a structured questionnaire to assess smoking status, physical activity, 
income, and educational and marital status. Smoking status was categorized into two groups: non-smokers or ex-smokers and current smokers. Regular physical activity was defined as exercising moderately for over $30 \mathrm{~min}$ per session more than 5 times per week or vigorously for over 20 min per session more than 3 times per week. Individuals with household incomes in the lowest quartile were designated as the low income group. Education level was classified as low if participants did not finish education higher than ninth grade. Daily energy intake was assessed using a single $24 \mathrm{~h}$ recall method.

\section{Statistical analysis}

All statistical analyses were performed using the SPSS complex sample procedure by reflecting strata, clusters and weighted values applied to the KNHANES. Comparisons of participant characteristics according to abdominal obesity by sex were conducted using Student's $t$ test for continuous variables and the $\chi^{2}$ test for categorical variables. We also used the $\chi^{2}$ test to analyse the frequency of drinking, drinking quantity and binge drinking pattern depending on abdominal obesity. Potential confounders in the analyses of alcohol drinking pattern variables were sex, age, BMI, education level, income, physical activity, smoking status and total energy intake. Prior to inclusion of the confounders, variance inflation factors were calculated and all were lower than 3 (data not shown). In addition, alcohol drinking frequency was a confounder in the analysis of typical drinking quantity and typical drinking quantity was a confounder in the analysis of alcohol drinking frequency. A $P$ value less than 0.05 was considered to be statistically significant. All analyses including calculation of weighted means, percentages, standard errors and odds ratios were conducted using the statistical software package IBM SPSS Statistics version 21.0.

\section{Results}

A total of 11289 normal-weight individuals were included in the present study and their general characteristics are presented in Table 1. Among the study participants, $7.9 \%$ ( $n 379$ ) of men and $7.6 \%$ ( $n$ 609) of women had abdominal obesity. Compared with normal-weight individuals without abdominal obesity, normal-weight individuals with abdominal obesity were older $(52 \cdot 13$ (sE 0.15) v. 55.39 (SE 0.48) years in males; 51.09 (SE 0.12) v. 57.64 (SE 0.38) years in females) and had higher BMI (22.46 (SE 0.03) $v$. 24.09 (SE 0.04 ) kg/m² in males; 22.20 (SE 0.02) v. 23.96 (SE $0.04 \mathrm{~kg} / \mathrm{m}^{2}$ ) in females). With respect to additional variables, normal-weight men with abdominal obesity were less likely to engage in regular physical activity, were less educated and had higher total energy intake. Normalweight women with abdominal obesity were less likely to be educated or married living with spouse, had lower income and had lower total energy intake. The overall percentage of normal-weight subjects with abdominal obesity who consumed 10 drinks or more per typical occasion was higher compared with those without abdominal obesity in both sexes (19.2 v. $15.7 \%$ in males; $5 \cdot 1$ v. $1.5 \%$ in females; Table 1$)$.

Multivariate logistic analysis was conducted to examine the relationship between drinking pattern and abdominal obesity in normal-weight persons (Table 2). Smoking status was included as a covariate because it has been found to be an important confounder of alcohol drinking pattern and abdominal fatness ${ }^{(27)}$. Compared with normal-weight men who consumed fewer than 2 drinks per typical occasion, those who consumed more than 7 drinks were more prone to have abdominal obesity $(\mathrm{OR}=2 \cdot 16$; $95 \%$ CI $1.23,3.77$ for $7-9$ drinks/typical occasion; OR =2.07; $95 \%$ CI 1.19, 3.59 for $\geq 10$ drinks/typical occasion) when adjusting for age, BMI, physical activity, smoking status, education, income and energy intake. These associations were still significant after controlling for drinking frequency $(\mathrm{OR}=2 \cdot 00 ; 95 \%$ CI 1.08, 3.66 for 7-9 drinks/typical occasion; OR =1.86; $95 \%$ CI 1.01, 3.41 for $\geq 10$ drinks/typical occasion). Normal-weight males who reported consuming $\geq 7$ standard drinks consecutively on one occasion at a frequency of almost every day were found to have a higher odds for abdominal obesity compared with non-binge drinkers (OR $=2 \cdot 10 ; 95 \%$ CI 1.21, 3.63; Table 3 ).

Normal-weight women who consumed more than 10 drinks per typical occasion were more likely to have abdominal obesity compared with women who consumed fewer than 2 drinks per typical occasion $(\mathrm{OR}=3 \cdot 26$; $95 \%$ CI 1.12 , 9.47) after adjusting for age, BMI, education, smoking status, income, physical activity and energy intake. This result was still significant after adjusting for drinking frequency $(\mathrm{OR}=3 \cdot 34 ; 95 \% \mathrm{CI} 1 \cdot 21,9 \cdot 18)$. Binge drinking in normal-weight women, defined as consuming $\geq 5$ standard drinks consecutively on one occasion at any frequency, was not a significant factor of abdominal obesity after adjusting for the variables listed above (Table 4).

With respect to abdominal obesity and alcohol drinking frequency, there was no significant association in either normal-weight males or normal-weight females (Tables 3 and 4).

Table 5 shows the quantitative effect of alcohol on abdominal obesity in normal-weight persons. Among participants consuming 21 or more drinks weekly, the odds for normal-weight abdominal obesity were 1.47 (95\% CI 1.07, 2.04) compared with those consuming 1-6 drinks weekly. A dose-response relationship was found between total alcohol intake and normal-weight abdominal obesity in both genders, without clinical significance after adjustment for confounders.

\section{Discussion}

Results from the current study revealed that both normalweight men and women who consumed a higher quantity 
Table 1 General characteristics of participants according to abdominal obesity* and sex; middle-aged adults (40-69 years), Korea National Health and Nutrition Examination Survey $2008-2013$

\begin{tabular}{|c|c|c|c|c|c|c|c|c|c|c|c|c|c|c|c|}
\hline & \multicolumn{5}{|c|}{ Male $(n$ 4491) } & \multicolumn{5}{|c|}{ Female $(n 6798)$} & \multicolumn{5}{|c|}{ Total ( $n$ 11289) } \\
\hline & \multicolumn{2}{|c|}{$\begin{array}{c}\text { Normal WC } \\
(n \text { 4112) }\end{array}$} & \multicolumn{2}{|c|}{$\begin{array}{l}\text { Abdominal } \\
\text { obesity } \\
(n 379)\end{array}$} & \multirow[b]{2}{*}{$P$ value } & \multicolumn{2}{|c|}{$\begin{array}{l}\text { Normal WC } \\
(n \text { 6189) }\end{array}$} & \multicolumn{2}{|c|}{$\begin{array}{c}\text { Abdominal } \\
\text { obesity } \\
(n 609)\end{array}$} & \multirow[b]{2}{*}{$P$ value } & \multicolumn{2}{|c|}{$\begin{array}{l}\text { Normal WC } \\
(n \text { 10301) }\end{array}$} & \multicolumn{2}{|c|}{$\begin{array}{l}\text { Abdominal } \\
\text { obesity } \\
(n 988)\end{array}$} & \multirow[b]{2}{*}{$P$ value } \\
\hline & $\begin{array}{c}\text { Mean or } \\
\%\end{array}$ & SE & $\begin{array}{c}\text { Mean or } \\
\%\end{array}$ & SE & & $\begin{array}{c}\text { Mean or } \\
\%\end{array}$ & SE & $\begin{array}{c}\text { Mean or } \\
\%\end{array}$ & SE & & $\begin{array}{c}\text { Mean or } \\
\%\end{array}$ & SE & $\begin{array}{c}\text { Mean or } \\
\%\end{array}$ & SE & \\
\hline WC (cm) & 80.5 & 0.1 & 91.9 & 0.1 & $<0.001$ & $75 \cdot 0$ & 0.1 & 87.5 & 0.1 & $<0.001$ & $76 \cdot 3$ & 0.1 & 89.6 & 0.1 & $<0.001$ \\
\hline Age (years) & $52 \cdot 1$ & 0.1 & 55.4 & 0.4 & $<0.001$ & $51 \cdot 1$ & 0.1 & $57 \cdot 6$ & 0.3 & $<0.001$ & $45 \cdot 0$ & 0.2 & 44.1 & 0.2 & $<0.001$ \\
\hline BMI $\left(\mathrm{kg} / \mathrm{m}^{2}\right)$ & $22 \cdot 4$ & 0.1 & $24 \cdot 1$ & $0 \cdot 1$ & $<0.001$ & $22 \cdot 2$ & 0.1 & 23.9 & $0 \cdot 1$ & $<0.001$ & $22 \cdot 1$ & 0.1 & 24.0 & 0.1 & $<0.001$ \\
\hline Smoking status (\% current smokers) & $44 \cdot 3$ & 1.0 & 41.4 & 3.3 & 0.355 & 4.5 & 0.3 & $6 \cdot 1$ & 1.3 & 0.212 & $23 \cdot 3$ & 0.6 & $23 \cdot 2$ & 1.9 & 0.945 \\
\hline Physical activity (\% regular physical activity)† & 24.8 & 0.8 & $17 \cdot 8$ & $2 \cdot 3$ & 0.009 & $21 \cdot 1$ & 0.6 & $20 \cdot 2$ & $2 \cdot 0$ & 0.672 & $23 \cdot 3$ & 0.6 & 19.1 & 1.5 & 0.013 \\
\hline Education (\% $\leq$ graduated middle school) & 34.8 & 0.9 & 41.0 & $3 \cdot 3$ & 0.049 & 38.0 & 0.9 & 72.9 & $2 \cdot 3$ & $<0.001$ & $36 \cdot 4$ & 0.8 & 57.5 & $2 \cdot 2$ & $<0.001$ \\
\hline Income (\% lowest quartile) $\ddagger$ & $26 \cdot 6$ & 0.9 & $26 \cdot 9$ & $2 \cdot 7$ & 0.905 & $22 \cdot 8$ & 0.7 & 28.4 & $2 \cdot 4$ & 0.020 & 24.6 & 0.6 & $27 \cdot 7$ & 1.8 & 0.096 \\
\hline Marital status (\% currently married living with spouse) & $89 \cdot 2$ & 0.6 & $92 \cdot 1$ & 1.8 & 0.128 & 83.9 & 0.6 & $77 \cdot 6$ & $2 \cdot 1$ & 0.001 & $86 \cdot 4$ & 0.5 & 84.7 & 1.5 & 0.218 \\
\hline Energy intake (kJ/d) & 9739.5 & $75 \cdot 7$ & 9864.6 & 225.5 & $<0.001$ & $7135 \cdot 4$ & $43 \cdot 1$ & $6695 \cdot 7$ & $120 \cdot 1$ & $<0.001$ & 8393.9 & $37 \cdot 2$ & $8434 \cdot 1$ & 38.9 & 0.385 \\
\hline Typical drinking quantity (drinks/typical occasion) & & & & & 0.138 & & & & & 0.057 & & & & & 0.004 \\
\hline $1-2$ & 20.5 & 0.8 & $14 \cdot 6$ & $2 \cdot 3$ & & $61 \cdot 1$ & 1.0 & 59.8 & 3.3 & & $39 \cdot 3$ & 0.7 & 33.0 & $2 \cdot 2$ & \\
\hline $3-4$ & $22 \cdot 0$ & 0.9 & 23.4 & $2 \cdot 9$ & & $25 \cdot 1$ & 0.8 & $22 \cdot 8$ & $2 \cdot 8$ & & $23 \cdot 4$ & 0.6 & $23 \cdot 2$ & $2 \cdot 0$ & \\
\hline $5-6$ & $22 \cdot 3$ & 0.9 & $19 \cdot 7$ & 3.0 & & $9 \cdot 0$ & 0.6 & 7.8 & 1.9 & & $16 \cdot 2$ & 0.5 & 14.9 & $2 \cdot 0$ & \\
\hline $7-9$ & 19.5 & 0.8 & $23 \cdot 1$ & 3.0 & & $3 \cdot 3$ & 0.4 & 4.5 & 1.4 & & $12 \cdot 0$ & 0.5 & $15 \cdot 5$ & 1.9 & \\
\hline$\geq 10$ & $15 \cdot 7$ & 0.8 & $19 \cdot 2$ & $2 \cdot 9$ & & 1.5 & 0.3 & $5 \cdot 1$ & $2 \cdot 0$ & & 9.1 & 0.4 & 13.4 & 1.9 & \\
\hline Alcohol drinking frequency in past year & & & & & 0.272 & & & & & 0.090 & & & & & 0.063 \\
\hline$<1 /$ month & $12 \cdot 8$ & 0.7 & 8.8 & $2 \cdot 0$ & & 41.0 & 0.9 & $47 \cdot 3$ & 3.5 & & $25 \cdot 9$ & 0.6 & 24.4 & $2 \cdot 1$ & \\
\hline $1 /$ month & $9 \cdot 0$ & 0.6 & 7.9 & 1.8 & & 18.6 & 0.7 & $15 \cdot 8$ & 2.5 & & $13 \cdot 4$ & 0.5 & 11.1 & 1.5 & \\
\hline $2-4 /$ month & $28 \cdot 1$ & 0.9 & $25 \cdot 5$ & $3 \cdot 0$ & & $26 \cdot 3$ & 0.9 & $22 \cdot 7$ & 2.7 & & $27 \cdot 3$ & 0.6 & 24.4 & $2 \cdot 1$ & \\
\hline 2-3/week & $30 \cdot 0$ & 1.0 & 34.0 & $3 \cdot 2$ & & $11 \cdot 2$ & 0.7 & 8.7 & $2 \cdot 0$ & & 21.3 & 0.6 & 23.8 & $2 \cdot 2$ & \\
\hline$\geq 4 /$ week & $20 \cdot 2$ & 0.8 & 23.8 & $3 \cdot 1$ & & 2.9 & 0.3 & 5.4 & 1.7 & & $12 \cdot 1$ & 0.5 & $16 \cdot 4$ & $2 \cdot 1$ & \\
\hline Binge drinking ( $\geq 7$ drinks (men), $\geq 5$ drinks (women)) & & & & & 0.120 & & & & & 0.367 & & & & & 0.035 \\
\hline None & $21 \cdot 2$ & 0.8 & $16 \cdot 6$ & $2 \cdot 3$ & & $50 \cdot 6$ & 1.0 & 53.5 & 3.5 & & 34.9 & 0.7 & 31.6 & $2 \cdot 2$ & \\
\hline$<1 /$ month & 17.5 & 0.8 & $14 \cdot 7$ & 2.6 & & 25.0 & 0.9 & 22.5 & 3.1 & & 21.0 & 0.6 & $17 \cdot 9$ & 1.9 & \\
\hline $1 /$ month & $18 \cdot 4$ & 0.8 & $17 \cdot 8$ & $2 \cdot 6$ & & $13 \cdot 2$ & 0.7 & $13 \cdot 7$ & 2.5 & & $15 \cdot 9$ & 0.5 & $16 \cdot 1$ & 1.9 & \\
\hline $1 /$ week & $30 \cdot 7$ & 0.9 & 34.4 & 3.5 & & $9 \cdot 4$ & 0.6 & 6.9 & 1.6 & & $20 \cdot 8$ & 0.6 & $23 \cdot 2$ & $2 \cdot 3$ & \\
\hline Every day & $12 \cdot 2$ & 0.7 & $16 \cdot 6$ & $2 \cdot 6$ & & 1.8 & 0.3 & 3.4 & 1.4 & & $7 \cdot 4$ & 0.4 & 11.2 & 1.7 & \\
\hline Total alcohol intake (drinks/week) & & & & & 0.273 & & & & & 0.236 & & & & & 0.042 \\
\hline$<7$ & $49 \cdot 0$ & 1.0 & 41.8 & 3.6 & & 88.8 & 0.7 & $87 \cdot 8$ & $2 \cdot 3$ & & 67.5 & 0.7 & $60 \cdot 4$ & $2 \cdot 6$ & \\
\hline $7-13$ & $18 \cdot 5$ & 0.8 & $19 \cdot 7$ & $2 \cdot 6$ & & $7 \cdot 8$ & 0.6 & $8 \cdot 6$ & 1.9 & & 13.5 & 0.5 & $15 \cdot 2$ & 1.8 & \\
\hline $14-20$ & 11.6 & 0.7 & 13.9 & $2 \cdot 6$ & & 1.6 & 0.2 & 0.5 & 0.3 & & 6.9 & 0.4 & 8.5 & 1.5 & \\
\hline$\geq 21$ & 20.9 & 0.9 & 24.5 & 3.2 & & 1.8 & 0.3 & $3 \cdot 1$ & 1.4 & & $12 \cdot 0$ & 0.5 & 15.9 & $2 \cdot 1$ & \\
\hline
\end{tabular}

WC, waist circumference.

sity is defined as WC $>90 \mathrm{~cm}$ for men and $>85 \mathrm{~cm}$ for women in accordance with the Korean Society for the Study of Obesity (25).

tRegular physical activity is defined as exercising moderately for $>30 \mathrm{~min} / \mathrm{session}$ for $>5$ times/week or vigorously for $>20 \mathrm{~min} / \mathrm{session}$ for $>3$ times/week.

Lowest level is defined as the lowest 25 percentile. 
Table 2 Associations between alcohol drinking pattern and normal-weight abdominal obesity; middle-aged adults (40-69 years), Korea National Health and Nutrition Examination Survey 2008-2013

\begin{tabular}{|c|c|c|c|c|c|c|}
\hline & \multicolumn{2}{|c|}{ Model $1^{*}$} & \multicolumn{2}{|c|}{ Model $2 \dagger$} & \multicolumn{2}{|c|}{ Model $3 \ddagger$} \\
\hline & OR & $95 \% \mathrm{Cl}$ & OR & $95 \% \mathrm{Cl}$ & OR & $95 \% \mathrm{Cl}$ \\
\hline \multicolumn{7}{|c|}{ Typical drinking quantity (drinks/typical occasion) } \\
\hline $1-2$ & 1.00 & Ref. & 1.00 & Ref. & 1.00 & Ref. \\
\hline $3-4$ & $1 \cdot 18$ & $0.91,1.53$ & 1.27 & $0.96,1.70$ & 1.35 & $0.99,1.85$ \\
\hline $5-6$ & $1 \cdot 10$ & $0.78,1.54$ & 1.16 & $0.81,1.66$ & $1 \cdot 19$ & $0.81,1.75$ \\
\hline $7-9$ & 1.54 & $1 \cdot 11,2 \cdot 13$ & 1.80 & $1 \cdot 19,2 \cdot 74$ & 1.88 & $1.19,2.97$ \\
\hline$\geq 10$ & 1.76 & $1 \cdot 26,2.46$ & 1.92 & $1 \cdot 28,2 \cdot 86$ & 1.96 & $1.25,3.02$ \\
\hline$P$ for linear trend§ & \multicolumn{2}{|c|}{0.138} & \multicolumn{2}{|c|}{0.042} & \multicolumn{2}{|c|}{0.061} \\
\hline \multicolumn{3}{|c|}{ Alcohol drinking frequency in past year } & & & & \\
\hline$<1 /$ month & 1.00 & Ref. & 1.00 & Ref. & 1.00 & Ref. \\
\hline $1 /$ month & 0.88 & $0.62,1.24$ & 0.91 & $0.63,1.34$ & 0.84 & $0.57,1.23$ \\
\hline $2-4 /$ month & 0.95 & $0.72,1.25$ & 0.93 & $0.68,1.26$ & 0.79 & $0.56,1.10$ \\
\hline 2-3/week & $1 \cdot 18$ & $0.89,1.57$ & $1 \cdot 14$ & $0.82,1.59$ & 0.90 & $0.61,1.32$ \\
\hline$\geq 4 /$ week & 1.43 & $1.01,2.03$ & $1 \cdot 26$ & $0.81,1.96$ & 0.95 & $0.59,1.54$ \\
\hline$P$ for linear trend§ & \multicolumn{2}{|c|}{0.077} & \multicolumn{2}{|c|}{0.449} & \multicolumn{2}{|c|}{0.582} \\
\hline \multicolumn{7}{|l|}{ Binge drinking\| } \\
\hline None & 1.00 & Ref. & 1.00 & Ref. & 1.00 & Ref. \\
\hline$<1 /$ month & 0.94 & $0.07,1.26$ & 1.02 & $0.73,1.41$ & 0.91 & $0.64,1.30$ \\
\hline $1 /$ month & 1.11 & $0.81,1.53$ & 1.21 & $0.86,1.71$ & 1.01 & $0.65,1.55$ \\
\hline 1/week & $1 \cdot 23$ & $0.93,1.63$ & $1 \cdot 20$ & $0.87,1.65$ & 0.87 & $0.53,1.43$ \\
\hline Every day & & & 1.86 & $1.16,2.99$ & 1.21 & $0.62,2.34$ \\
\hline$P$ for linear trend $\S$ & \multicolumn{2}{|c|}{$0.022^{1.15,2.43}$} & \multicolumn{2}{|c|}{0.005} & \multicolumn{2}{|c|}{0.123} \\
\hline
\end{tabular}

Ref., reference category.

*Unadjusted.

†Adjusted for sex, age, BMI, physical activity, smoking status, education, income and energy intake.

$\ddagger$ Adjusted for all variables in Model 2 and other alcohol drinking pattern (typical drinking quantity or alcohol drinking frequency).

$\S$ Significance of $P$ for trend by multiple logistic regression analysis.

\Binge drinking is defined as consuming $\geq 7$ drinks (men) or $\geq 5$ drinks (women) consecutively on one occasion.

Table 3 Associations between alcohol drinking pattern and abdominal obesity in normal-weight, middle-aged men (40-69 years); Korea National Health and Nutrition Examination Survey 2008-2013

\begin{tabular}{|c|c|c|c|c|c|c|}
\hline & \multicolumn{2}{|c|}{ Model $1^{*}$} & \multicolumn{2}{|c|}{ Model $2 \dagger$} & \multicolumn{2}{|c|}{ Model 3‡ } \\
\hline & OR & $95 \% \mathrm{Cl}$ & OR & $95 \% \mathrm{Cl}$ & OR & $95 \% \mathrm{Cl}$ \\
\hline \multicolumn{7}{|c|}{ Typical drinking quantity (drinks/typical occasion) } \\
\hline $1-2$ & 1.00 & Ref. & 1.00 & Ref. & 1.00 & Ref. \\
\hline $3-4$ & 1.49 & $0.96,2.32$ & 1.59 & $0.96,2.61$ & 1.53 & $0.89,2.64$ \\
\hline $5-6$ & 1.24 & $0.76,2.01$ & 1.31 & $0.77,2.22$ & 1.23 & $0.69,2.21$ \\
\hline $7-9$ & 1.66 & $1.05,2.63$ & $2 \cdot 16$ & $1.23,3.77$ & 2.00 & $1.08,3.66$ \\
\hline$\geq 10$ & 1.72 & $1.06,2.78$ & 2.07 & $1.19,3.59$ & 1.86 & $1.01,3.41$ \\
\hline$P$ for linear trend§ & \multicolumn{2}{|c|}{0.224} & \multicolumn{2}{|c|}{0.057} & \multirow{2}{*}{\multicolumn{2}{|c|}{0.156}} \\
\hline Alcohol drinking fre & past $\mathrm{y}$ & & & & & \\
\hline$<1 /$ month & 1.00 & Ref. & 1.00 & Ref. & 1.00 & Ref. \\
\hline $1 /$ month & 1.27 & $0.64,2.51$ & 1.20 & $0.57,2.50$ & 1.05 & $0.49,2.25$ \\
\hline $2-4 /$ month & 1.32 & $0.75,2.29$ & 1.15 & $0.61,2.15$ & 0.94 & $0.48,1.84$ \\
\hline 2-3/week & 1.64 & $0.96,2.80$ & 1.59 & $0.87,2.89$ & 1.23 & $0.63,2.39$ \\
\hline$\geq 4 /$ week & 1.71 & $0.97,3.03$ & 1.63 & $0.84,3.15$ & 1.23 & $0.60,2.53$ \\
\hline$P$ for linear trend§ & \multicolumn{2}{|c|}{0.191} & \multicolumn{2}{|c|}{0.137} & \multicolumn{2}{|c|}{0.323} \\
\hline \multicolumn{7}{|l|}{ Binge drinking $\|$} \\
\hline None & 1.00 & Ref. & 1.00 & Ref. & 1.00 & Ref. \\
\hline$<1 /$ month & 1.06 & $0.65,1.74$ & 0.93 & $0.55,1.59$ & 0.80 & $0.44,1.45$ \\
\hline $1 /$ month & 1.24 & $0.78,1.94$ & 1.27 & $0.77,2.08$ & 1.02 & $0.56,1.88$ \\
\hline $1 /$ week & 1.43 & $0.95,2.14$ & 1.38 & $0.88,2.17$ & 0.97 & $0.48,1.96$ \\
\hline Every day & 1.73 & $1.09,2 \cdot 72$ & $2 \cdot 10$ & $1.21,3.63$ & 1.44 & $0.64,3.25$ \\
\hline$P$ for linear trend§ & \multicolumn{2}{|c|}{$0.150^{1 \cdot 09,2 \cdot 12}$} & \multicolumn{2}{|c|}{0.002} & \multicolumn{2}{|c|}{0.053} \\
\hline
\end{tabular}

Ref., reference category.

*Unadjusted.

†Adjusted for age, BMI, physical activity, smoking status, education, income and energy intake.

$\ddagger$ Adjusted for all variables in Model 2 and other alcohol drinking pattern (typical drinking quantity or alcohol drinking frequency).

$\S$ Significance of $P$ for trend by multiple logistic regression analysis.

||Binge drinking is defined as consuming $\geq 7$ drinks consecutively on one occasion. 
Table 4 Associations between alcohol drinking pattern and abdominal obesity in normal-weight, middle-aged women (40-69 years); Korea National Health and Nutrition Examination Survey 2008-2013

\begin{tabular}{|c|c|c|c|c|c|c|}
\hline & \multicolumn{2}{|c|}{ Model $1^{*}$} & \multicolumn{2}{|c|}{ Model $2 \dagger$} & \multicolumn{2}{|c|}{ Model 3‡ } \\
\hline & OR & $95 \% \mathrm{Cl}$ & OR & $95 \% \mathrm{Cl}$ & OR & $95 \% \mathrm{Cl}$ \\
\hline \multicolumn{7}{|c|}{ Typical drinking quantity (drinks/typical occasion) } \\
\hline $1-2$ & 1.00 & Ref. & 1.00 & Ref. & 1.00 & Ref. \\
\hline $3-4$ & 0.93 & $0.66,1.30$ & 1.19 & $0.81,1.76$ & 1.32 & $0.88,1.97$ \\
\hline $5-6$ & 0.88 & $0.52,1.51$ & 1.42 & $0.82,2.48$ & 1.32 & $0.75,2.31$ \\
\hline $7-9$ & 1.38 & $0.68,2.78$ & 1.78 & $0.77,4.14$ & 1.79 & $0.76,4.26$ \\
\hline$\geq 10$ & 3.37 & $1.41,8.06$ & 3.26 & $1.12,9.47$ & 3.34 & $1 \cdot 21,9 \cdot 18$ \\
\hline$P$ for linear trend§ & \multicolumn{2}{|c|}{0.206} & \multicolumn{2}{|c|}{0.182} & \multicolumn{2}{|c|}{0.268} \\
\hline \multicolumn{3}{|c|}{ Alcohol drinking frequency in past year } & & & & \\
\hline$<1 /$ month & 1.00 & Ref. & 1.00 & Ref. & 1.00 & Ref. \\
\hline $1 /$ month & 0.73 & $0.49,1 \cdot 10$ & 0.84 & $0.83,1.32$ & 0.79 & $0.50,1.26$ \\
\hline $2-4 /$ month & 0.75 & $0.53,1.05$ & 0.93 & $0.63,1.35$ & 0.82 & $0.55,1.23$ \\
\hline 2-3/week & 0.67 & $0.39,1.15$ & 0.78 & $0.41,1.48$ & 0.66 & $0.34,1.29$ \\
\hline$\geq 4 /$ week & 1.63 & $0.81,3.29$ & 1.70 & $0.67,4.29$ & 1.37 & $0.55,3.45$ \\
\hline$P$ for linear trend§ & \multicolumn{2}{|c|}{0.347} & \multicolumn{2}{|c|}{0.580} & \multicolumn{2}{|c|}{0.739} \\
\hline \multicolumn{7}{|l|}{ Binge drinking\| } \\
\hline None & 1.00 & Ref. & 1.00 & Ref. & 1.00 & Ref. \\
\hline$<1 /$ month & 0.85 & $0.59,1.23$ & $1 \cdot 15$ & $0.77,1.71$ & 0.98 & $0.65,1.48$ \\
\hline $1 /$ month & 0.99 & $0.63,1.53$ & 1.23 & $0.77,1.98$ & 0.95 & $0.53,1.69$ \\
\hline 1/week & 0.69 & $0.41,1.17$ & 0.82 & $0.43,1.56$ & 0.58 & $0.26,1.28$ \\
\hline Every day & 1.78 & $0.73,4.33$ & 1.78 & $0.53,6.03$ & 0.75 & $0.15,3.72$ \\
\hline$P$ for linear trend§ & \multicolumn{2}{|c|}{$0.142^{0.13,4.30}$} & \multicolumn{2}{|c|}{$0.535^{0.53,0.03}$} & \multicolumn{2}{|c|}{0.979} \\
\hline
\end{tabular}

Ref., reference category.

*Unadjusted.

†Adjusted for age, BMI, physical activity, smoking status, education, income and energy intake.

$\ddagger$ Adjusted for all variables in Model 2 and other alcohol drinking pattern (typical drinking quantity or alcohol drinking frequency).

$\S$ Significance of $P$ for trend by multiple logistic regression analysis.

$\|$ Binge drinking is defined as consuming $\geq 5$ drinks consecutively on one occasion.

of alcohol on each drinking occasion had higher odds for abdominal obesity compared with individuals who consumed fewer than 2 drinks, even after controlling for alcohol drinking pattern. Normal-weight men who consumed $\geq 7$ standard drinks on one occasion were at significantly increased risk of abdominal obesity, while normal-weight women were at significantly increased risk with consumption of $\geq 10$ standard drinks on one occasion. In normal-weight males, binge drinking $(\geq 7$ standard drinks consecutively) every day was associated with higher odds of abdominal obesity. However, this association was not significant in female, normal-weight, binge drinkers (defined as $\geq 5$ standard drinks consecutively on one occasion). In contrast, alcohol drinking frequency was not significantly associated with abdominal obesity in both genders or overall.

Although it is widely recognized that alcohol intake contributes to the risk of obesity, previous studies on the relationship between alcohol intake and obesity have reported inconsistent findings ${ }^{(21,28,29)}$. An observational study using the same database of the general Korean population as the present study revealed that there were positive relationships between heavy drinking, binge drinking and abdominal obesity ${ }^{(30)}$. In one study in Finland, women who did not drink alcohol had the highest risk of obesity ${ }^{(31)}$, while another study reported no relationship between BMI and alcohol intake in men ${ }^{(32)}$. In contrast, Riserus et al. showed that high alcohol intake was associated with abdominal obesity rather than generalized obesity $^{(28)}$, in accordance with abundant cross-sectional data from studies conducted in other countries among young, middle-aged and elderly men and women ${ }^{(33-35)}$. These studies reported an association between high alcohol intake and abdominal obesity only in individuals in the lowest tertile of BMI, which supports an effect of alcohol on abdominal fat accumulation per $s e^{(28)}$, in agreement with our findings. Evidence from some previous studies showed that heavy drinkers have low BMI combined with high WC or WHR compared with healthy social drinkers ${ }^{(28,36)}$.

One possible explanation for the effect of alcohol on abdominal obesity is an increased energy intake through high alcohol intake, resulting in fat accumulation ${ }^{(37)}$. However, contrary to all other energy sources, alcohol cannot be stored in the body, with its absolute metabolic priority ${ }^{(38)}$. Thus a non-caloric mechanism must be taken into consideration for alcohol to contribute to abdominal obesity. Endocrine changes due to alcohol intake seem to be of importance. Alcohol alters steroid metabolism in the liver, which subsequently induces fat accumulation ${ }^{(39)}$. With activation of the hypothalamus-pituitary-adrenal axis, alcohol consumption leads to the release of cortisol, resulting in suppression of fat oxidation with accumulation of mono-oxidized fat in the abdomen ${ }^{(40)}$. Also, alcohol has catabolic effects on muscle by affecting levels of anabolic sex hormones and growth hormone, which are necessary 
for preserving muscle mass ${ }^{(28)}$. Through these mechanisms, abdominal obesity increases fat deposition in the liver and muscles or around vessels and induces insulin resistance, leading to CVD such as hypertension, diabetes and dyslipidaemia following atherosclerosis ${ }^{(10)}$. Furthermore, abdominal adiposity in normal-weight individuals may imply excess fat in the trunk, representing relatively lower appendicular skeletal muscle mass, which is known as sarcopenic obesity. Both central obesity and sarcopenic obesity are known to be strongly associated with metabolic risk $^{(41)}$. Therefore, normal-weight persons with increased abdominal fat deposition are at risk of developing an adverse metabolic profile.

In our study, alcohol drinking frequency had no significant association with abdominal obesity in normalweight subjects. A few studies have suggested that drinking frequency was inversely associated with high $\mathrm{BMI}^{(18,42)}$. Another finding in a representative Korean population showed that drinking frequency was not associated with prevalence of metabolic syndrome ${ }^{(26)}$. There is a scarcity of evidence on the relationship between abdominal obesity and alcohol drinking frequency conducted in normal-weight subjects. Increased thermogenesis by alcohol consumption may be the biological mechanism behind the results in the present study. Alcohol metabolism uses three different pathways: the alcohol dehydrogenase pathway, the microsomal ethanoloxidizing system and oxidation by catalase ${ }^{(38)}$. Although alcohol is mostly degraded by the alcohol dehydrogenase pathway, frequent alcohol drinking also activates the microsomal ethanol-oxidizing system, resulting in increased energy consumption through its thermogenic effect ${ }^{(18)}$. This effect may be in balance with various metabolic alterations induced by alcohol consumption and additional energy from alcohol intake ${ }^{(18)}$. Therefore, alcohol drinking frequency may not be causally related to body weight or fat accumulation; rather, high alcohol intake over the threshold based on sex may have an effect on abdominal obesity in normal-weight individuals.

There are several limitations in our study. First, due to the cross-sectional study design, it is difficult to infer causality. Also, the potential for reverse causality may be found. It is plausible that a person with abdominal obesity was affected by alcohol drinking habit, such as underestimating his/her alcohol consumption or stopping drinking alcohol in order to reduce energy intake. Second, because alcohol drinking pattern was measured by selfreport on questionnaires, it is subject to reporting bias. As KNHANES does not have any procedure in the data collection to avoid this possibility except for initial validation of the study protocol, it may underestimate the effect size of the association in our study. In addition, we could not identify which alcoholic beverages specially promote abdominal fat deposition, because the types of alcoholic beverage were not classified. Third, a single day's dietary recall method in our study limits day-to-day variability in 
intake. The availability of multiple $24 \mathrm{~h}$ recalls will provide a more accurate assessment of usual intake at the individual level. Fourth, as our study combined participants from three waves of KNHANES, there might be a chance to include overlapping of some study subjects. However, there is little possibility of this type of bias because the survey is cross-checking the repeated cluster sampling. Finally, due to lack of imaging data of adipose tissue, information on body fat distribution was based on anthropometric indicators, such as WC, alone. However, despite these limitations, the current study examined whether alcohol drinking patterns are associated with abdominal obesity in normal-weight adults using the largest representative database of the general Korean population. In addition, adjustment for several important confounders including other drinking pattern and energy intake is another strength of the present study.

\section{Conclusion}

Overall, the amount of alcohol drinking per occasion was significantly associated with abdominal obesity in normalweight individuals aged 40-69 years in both sexes. Consumption of 7 or more standard drinks per occasion was positively associated with normal-weight abdominal obesity in middle-aged men; 10 or more standard drinks per occasion in middle-aged women. No significant relationship was found between alcohol drinking frequency and normal-weight abdominal obesity. Male every-day binge drinkers with normal weight had higher odds of abdominal obesity; there was no significant association in women. It has been suggested that normal-weight persons with abdominal obesity may represent an important target population for lifestyle modification and other preventive strategies ${ }^{(21)}$, and our results are consistent with data indicating increased risk of abdominal obesity in normalweight drinkers with a certain alcohol drinking pattern. Given that BMI may not be an accurate measurement of obesity, especially in persons with higher body fat and normal BMI ${ }^{(43)}$, we recommend that clinicians utilize other measurements of central adiposity such as WHR or WC, in addition to BMI, to diagnose obesity and predict obesityrelated cardiovascular risks. Lifestyle interventions including proper drinking guidelines and management of obesity-related health risks may be necessary in normalweight individuals with abdominal obesity.

\section{Acknowledgements}

Financial support: This research received no specific grant from any funding agency in the public, commercial or notfor-profit sectors. Conflict of interest: None. Authorship: All authors (K.-Y.P., H.-K.P. and H.-S.H.) contributed to the design of the study, interpretation of the results and development of the final manuscript. Ethics of buman subject participation: This study was conducted according to the guidelines laid down in the Declaration of Helsinki and all procedures involving human subjects were approved by the Institutional Review Board on Human Subjects Research and the Ethics Committee of Hanyang University Hospital. Written informed consent was obtained from all subjects.

\section{References}

1. World Health Organization (2000) Obesity: Preventing and Managing the Global Epidemic. Report of a WHO Consultation. WHO Technical Report Series no. 894. Geneva: WHO.

2. US Preventive Services Task Force (2003) Screening for obesity in adults: recommendations and rationale. Ann Intern Med 139, 930-932.

3. National Institute for Health and Clinical Excellence (2006) Obesity: Guidance on the Prevention, Identification, Assessment and Management of Overweight and Obesity in Adults and Children. NICE Clinical Guideline no. 43. London: NICE.

4. Whitlock G, Lewington S, Sherliker P et al. (2009) Bodymass index and cause-specific mortality in 900000 adults: collaborative analyses of 57 prospective studies. Lancet 373, 1083-1096.

5. Vanltallie TB (1998) Waist circumference: a useful index in clinical care and health promotion. Nutr Rev 56, 300-302.

6. Reis JP, Araneta MR, Wingard DL et al. (2009) Overall obesity and abdominal adiposity as predictors of mortality in US white and black adults. Ann Epidemiol 19, 134-142.

7. Rothman KJ (2008) BMI-related errors in the measurement of obesity. Int J Obes (Lond) 32, Suppl. 3, S56-S59.

8. Bigaard J, Frederiksen K, Tjonneland A et al. (2005) Waist circumference and body composition in relation to all-cause mortality in middle-aged men and women. Int J Obes (Lond) 29, 778-784.

9. Lee CMY, Huxley RR, Wildman RP et al. (2008) Indices of abdominal obesity are better discriminators of cardiovascular risk factors than BMI: a meta-analysis. J Clin Epidemiol 61, 646-653.

10. Sahakyan KR, Somers VK, Rodriguez-Escudero JP et al. (2015) Normal-weight central obesity: implications for total and cardiovascular mortality. Ann Intern Med 163, $827-835$.

11. Romero-Corral A, Somers VK, Sierra-Johnson J et al. (2009) Normal weight obesity: a risk factor for cardiometabolic dysregulation and cardiovascular mortality. Eur Heart J 31, 737-746.

12. Karter A, D'Agostino R, Mayer-Davis E et al. (2005) Abdominal obesity predicts declining insulin sensitivity in non-obese normoglycaemics: the Insulin Resistance Atherosclerosis Study (IRAS). Diabetes Obes Metab 7, 230-238.

13. Koh-Banerjee P, Chu N-F, Spiegelman D et al. (2003) Prospective study of the association of changes in dietary intake, physical activity, alcohol consumption, and smoking with 9-y gain in waist circumference among 16587 US men. Am J Clin Nutr 78, 719-727.

14. World Health Organization (1998) Obesity: Preventing and Managing the Global Epidemic. Report of a WHO Consultation on Obesity, Geneva, 3-5 June 1997. Geneva: WHO.

15. Traversy G \& Chaput JP (2015) Alcohol consumption and obesity: an update. Curr Obes Rep 4, 122-130.

16. Vadstrup ES, Petersen L, Sørensen TIA et al. (2003) Waist circumference in relation to history of amount and type of alcohol: results from the Copenhagen City Heart Study. Int J Obes Relat Metab Disord 27, 238-246. 
17. Lourenço S, Oliveira A \& Lopes C (2012) The effect of current and lifetime alcohol consumption on overall and central obesity. Eur J Clin Nutr 66, 813-818.

18. Tolstrup JS, Heitmann BL, Tjonneland AM et al. (2005) The relation between drinking pattern and body mass index and waist and hip circumference. Int J Obes (Lond) 29, 490-497.

19. Chakraborty S (2014) Analysis of NHANES 1999-2002 data reveals noteworthy association of alcohol consumption with obesity. Ann Gastroenterol 27, 250-257.

20. Halkjaer J, Tjonneland A, Thomsen BL et al. (2006) Intake of macronutrients as predictors of 5 -y changes in waist circumference. Am J Clin Nutr 84, 789-797.

21. MacInnis RJ, Hodge AM, Dixon HG et al. (2014) Predictors of increased body weight and waist circumference for middle-aged adults. Public Health Nutr 17, 1087-1097.

22. Mehta NK \& Chang VW (2009) Mortality attributable to obesity among middle-aged adults in the United States. Demography 46, 851-872.

23. Kim Y (2014) The Korea National Health and Nutrition Examination Survey (KNHANES): current status and challenges. Epidemiol Health 36, e2014002.

24. Weisell RC (2002) Body mass index as an indicator of obesity. Asia Pac J Clin Nutr 11, S681-S684.

25. Lee S, Park HS, Kim SM et al. (2006) Cut-off points of waist circumference for defining abdominal obesity in the Korean population. Korean J Obes 15, 1-9.

26. Lee K (2012) Gender-specific relationships between alcohol drinking patterns and metabolic syndrome: the Korea National Health and Nutrition Examination Survey 2008. Public Health Nutr 15, 1917-1924.

27. Travier N, Agudo A, May AM et al. (2009) Smoking and body fatness measurements: a cross-sectional analysis in the EPIC-PANACEA study. Prev Med 49, 365-373.

28. Riserus U \& Ingelsson E (2007) Alcohol intake, insulin resistance, and abdominal obesity in elderly men. Obesity (Silver Spring) 15, 1766-1773.

29. Suter PM (2005) Is alcohol consumption a risk factor for weight gain and obesity? Crit Rev Clin Lab Sci 42, 197-227.

30. Kim HN \& Song SW (2014) Relationships of both heavy and binge alcohol drinking with unhealthy habits in Korean adults based on the KNHANES IV data. Iran J Public Health 43, 579-589.

31. Lahti-Koski M, Pietinen P, Heliovaara M et al. (2002) Associations of body mass index and obesity with physical activity, food choices, alcohol intake, and smoking in the 1982-1997 FINRISK Studies. Am J Clin Nutr 75, 809-817.

32. Colditz GA, Giovannucci E, Rimm EB et al. (1991) Alcohol intake in relation to diet and obesity in women and men. Am J Clin Nutr 54, 49-55.

33. Dallongeville J, Marecaux N, Ducimetiere P et al. (1998) Influence of alcohol consumption and various beverages on waist girth and waist-to-hip ratio in a sample of French men and women. Int J Obes Relat Metab Disord 22, 1178-1183.

34. Leite ML \& Nicolosi A (2006) Lifestyle correlates of anthropometric estimates of body adiposity in an Italian middle-aged and elderly population: a covariance analysis. Int J Obes (Lond) 30, 926-934.

35. Lapidus L, Bengtsson C, Bergfors E et al. (2005) Alcohol intake among women and its relationship to diabetes incidence and all-cause mortality: the 32-year follow-up of a population study of women in Gothenburg, Sweden. Diabetes Care 28, 2230-2235.

36. Addolorato G, Capristo E, Greco AV et al. (1998) Influence of chronic alcohol abuse on body weight and energy metabolism: is excess ethanol consumption a risk factor for obesity or malnutrition? J Intern Med 244, 387-395.

37. Schroder H, Morales-Molina JA, Bermejo S et al. (2007) Relationship of abdominal obesity with alcohol consumption at population scale. Eur J Nutr 46, 369-376.

38. Suter PM, Hasler E \& Vetter W (1997) Effects of alcohol on energy metabolism and body weight regulation: is alcohol a risk factor for obesity? Nutr Rev 55, 157-171.

39. Bjorntorp P (1995) Endocrine abnormalities of obesity. Metabolism 44, 21-23.

40. Suter PM, Schutz Y \& Jequier E (1992) The effect of ethanol on fat storage in healthy subjects. $N$ Engl J Med 326, 983-987.

41. Despres JP (1998) The insulin resistance-dyslipidemic syndrome of visceral obesity: effect on patients' risk. Obes Res 6, Suppl. 1, 8S-17S.

42. Dorn JM, Hovey K, Muti P et al. (2003) Alcohol drinking patterns differentially affect central adiposity as measured by abdominal height in women and men. J Nutr $\mathbf{1 3 3}$, $2655-2662$.

43. Okorodudu DO, Jumean MF, Montori VM et al. (2010) Diagnostic performance of body mass index to identify obesity as defined by body adiposity: a systematic review and meta-analysis. Int J Obes (Lond) 34, 791-799. 